\title{
The psychological subtype of intimate partner violence and its effect on mental health: protocol for a systematic review and meta-analysis
}

Sarah Dokkedahl ${ }^{1 *}$ (D), Robin Niels Kok ${ }^{2,3}$, Siobhan Murphy', Trine Rønde Kristensen ${ }^{4}$, Ditte Bech-Hansen ${ }^{1}$ and Ask Elklit ${ }^{1}$

\begin{abstract}
Background/aim: Psychological violence is estimated to be the most common form of intimate partner violence (IPV). Despite this, research on the independent effect of psychological violence on mental health is scarce. Moreover, the lack of a clear and consistent definition of psychological violence has made results difficult to compare. The present study therefore aims to consolidate knowledge on psychological violence by conducting a systematic review and random-effects meta-analysis on the association between psychological violence and mental health problems, when controlling for other types of violence (e.g. physical and sexual) and taking into account severity, frequency, and duration of psychological violence.

Method: The present study is registered in the International Prospective Register for Systematic Reviews (PROSPERO; \#CRD42018116026) and the study design follows the Preferred Reporting Items for Systematic Reviews and Meta-Analyses (PRISMA; Additional file 1). A dual search will be conducted in the electronic databases PsycINFO, PubMed, EMBASE, and Web of Science. Data will be extracted using Endnote and Covidence and a meta-analysis will be conducted using Metafor-package in the programming language R. The Quality Assessment Tool for Quantitative Studies developed by the Effective Public Health Practice Project will be used to assess the quality of the included studies (i.e. weak, moderate and strong).

Results and discussion: The present review will help consolidate knowledge on psychological violence by evaluating whether frequency, severity or actual "type" of psychological violence produces the most harm. A thorough quality assessment will help overcome potential limitations regarding expected variations in terminology and assessment of psychological violence.

Systematic review registration: PROSPERO CRD42018116026.

Keywords: Psychological violence, Aggression, Coercion, Emotional abuse, Intimate partner violence, Mental health, PTSD, Psychometrics, Assessment, Abbreviations, DSM Diagnostic and statistical manual of mental disorders, EIGE European Institute of Gender Equality, ICD International Classification of Diseases, IPV Intimate Partner Violence, NICE National Institute for Health and Care Excellence, PMWI Psychological Maltreatment of Women Inventory, PTSD Posttraumatic Stress Disorder
\end{abstract}

\footnotetext{
* Correspondence: sdokkedahl@health.sdu.dk

${ }^{1}$ National Centre of Psychotraumatology, Department of Psychology at the

University of Southern Denmark, 5230 Odense M, Denmark

Full list of author information is available at the end of the article
}

(c) The Author(s). 2019 Open Access This article is distributed under the terms of the Creative Commons Attribution 4.0 International License (http://creativecommons.org/licenses/by/4.0/), which permits unrestricted use, distribution, and reproduction in any medium, provided you give appropriate credit to the original author(s) and the source, provide a link to the Creative Commons license, and indicate if changes were made. The Creative Commons Public Domain Dedication waiver (http://creativecommons.org/publicdomain/zero/1.0/) applies to the data made available in this article, unless otherwise stated. 


\section{Background}

Intimate partner violence (IPV) is a global health problem characterized as any behaviour within an intimate relationship that causes physical, psychological or sexual harm [1]. At present, it is well-documented that IPV can cause extensive mental health consequences among its victims [2-5]. IPV can be characterized as an interpersonal trauma, and symptoms of posttraumatic stress disorder (PTSD) have been identified in $31-84.4 \%$ of women exposed to IPV [2]; along with other comorbid symptoms such as depression, anxiety, suicidality, substance abuse and sleep disturbances $[2,5,6]$.

The subtype of psychological violence (compared to physical and sexual violence) is estimated to be the most common form of IPV in both the USA [7] and Europe [8], affecting between 35 and 49\% of men and women. This has led legislators in some European countries to criminalize psychological violence as an independent offence, making it equally punishable as physical violence (e.g. Norway [9] and England [10]). Although some researchers have argued that psychological violence in itself cannot be classified as a trauma, as it does not meet the first criterion of diagnosing PTSD (i.e. threat to life or physical integrity $[11,12])$, a more recent review on IPV and mental health argues that psychological violence can independently cause PTSD, depression and anxiety [13].

Despite both legal recognition of psychological violence and documentation of its effect on mental health, the conceptualization of the phenomenon is ambiguous in both research and clinical practice. Acts of psychological violence are distributed along a continuum starting from what is commonly termed psychological aggression (e.g. yelling and insults) and ending with more severe abuse, often labelled coercion (e.g. threats and isolation). How we interpret psychological aggression and how we distinguish it from more severe abuse depends, among other things, on the context in which it occurs, when it occurred in a sequence, how it was interpreted and whether it was perceived as abusive [11].

Another challenge is that psychological violence is often characterized in different ways. For example, the conceptualization of "coercive control" can generally be understood in two ways: firstly, as an overall attempt to control one's partner, in which IPV is a way of achieving control; secondly, as a subtype of IPV which is similar to-or a part of-the concept of psychological violence. The former is described in a structural perspective as more severe and gender-asymmetrical and is generally understood to be a representation of gender inequality [14-18]. The latter reflects a continuum of IPV from psychological aggression to more controlling behaviours constituting an assault [11]. Moreover, these are theoretical distinctions that have proven difficult for researchers and practitioners to conceptualize and apply.

The distinction between psychological violence and coercion is evident from the WHO, who define psychological violence (i.e. emotional or psychological abuse) such as "insults, belittling, constant humiliation, intimidation (e.g. destroying things), threats of harm, threats to take away children", while controlling behaviour is defined as "isolating a person from family and friends; monitoring their movements; and restricting access to financial resources, employment, education or medical care" [1] (p. 1). The specific acts of violence separate these two definitions, while the overall psychological harm combines them. Likewise, the European Institute of Gender Equality (EIGE) states an overall definition of psychological violence, which can be understood from the perspective of caused harm: "Any act or behaviour which causes psychological harm to the partner or former partner. Psychological violence can take the form of, among others, coercion, defamation, a verbal insult or harassment"19 (p. 45).

These variations in terminology are inevitably reflected in the psychometric instruments developed to assess psychological violence (Table 1; identified by the Centers for Disease Control and Prevention and the National Unit [20] against IPV in Denmark, LUV [21]). Indeed, the varying use of subscales (e.g. \#7 and \#9) and differing definitions of psychological violence make the findings from these studies difficult to compare and stresses the need to evaluate how the effect on mental health is influenced by such variations. For example, some instruments assess psychological aggression (e.g. \#10), while others measure more severe controlling behaviours (e.g. \#4). Furthermore, other instruments focus less on the act of violence and more on the effect on the victim (e.g. \#12). When measuring the effect of psychological violence on mental health, the difference between acts and effects can be crucial. Evidence demonstrates that some acts defined as psychologically violent (i.e. threats to kill/ harm) have been found to significantly load on a physical violence factor, most likely due to the aspect of physical threat, which makes the psychological and physical aspect hard to differentiate [11]. Furthermore, a majority of these assessment tools make use of frequency scores that do not differentiate between the severity of items. As such, items of psychological aggression (i.e. being called "ugly" and "worthless") are equated to severe controlling behaviour and threats (i.e. threats to kill or take away children). This exemplifies how important these distinctions are if we wish to understand the independent effect of psychological violence on mental health. Examining mental health while evaluating the instruments used to measure the phenomenon will help us understand whether severity, frequency or actual 


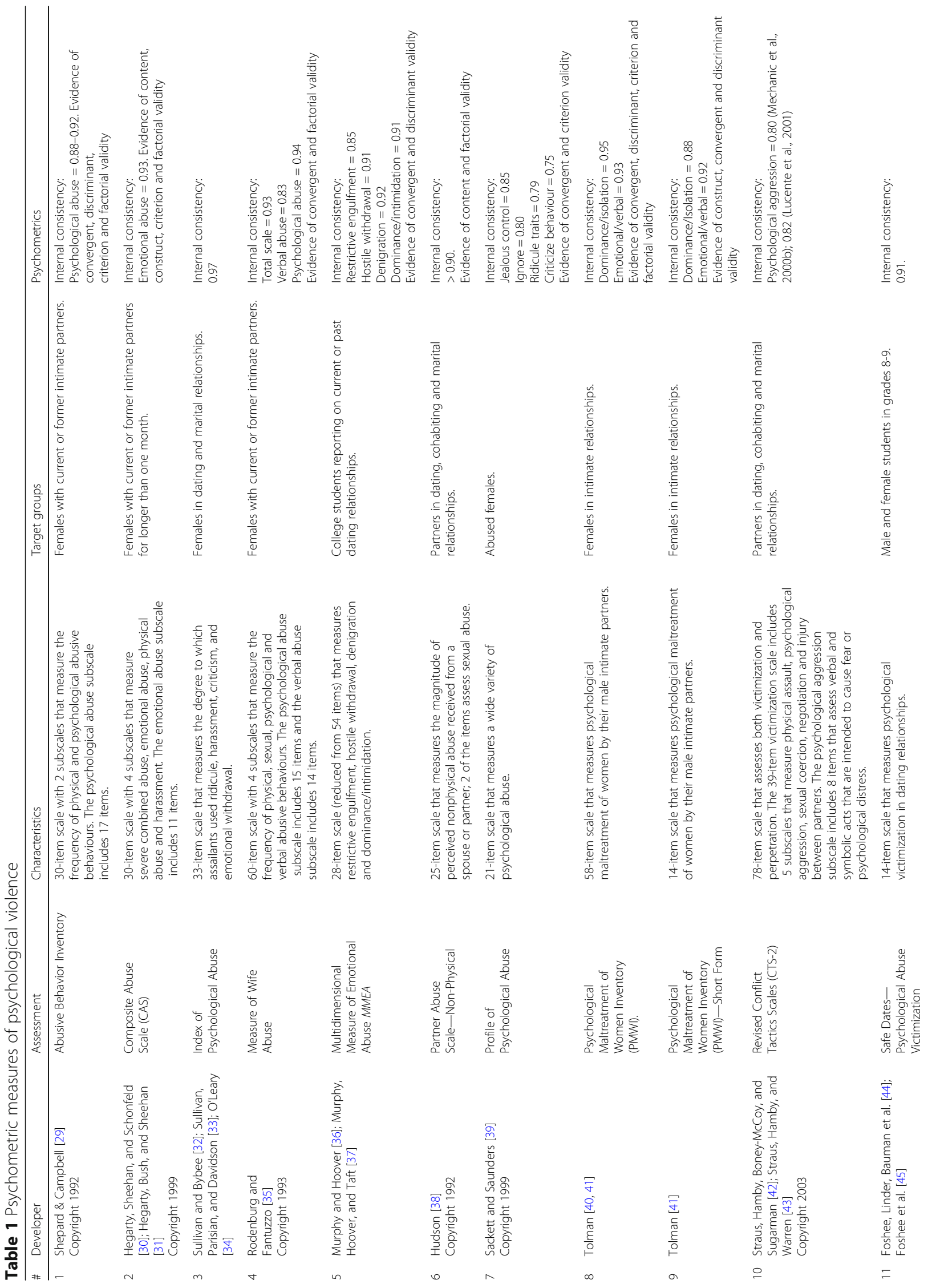




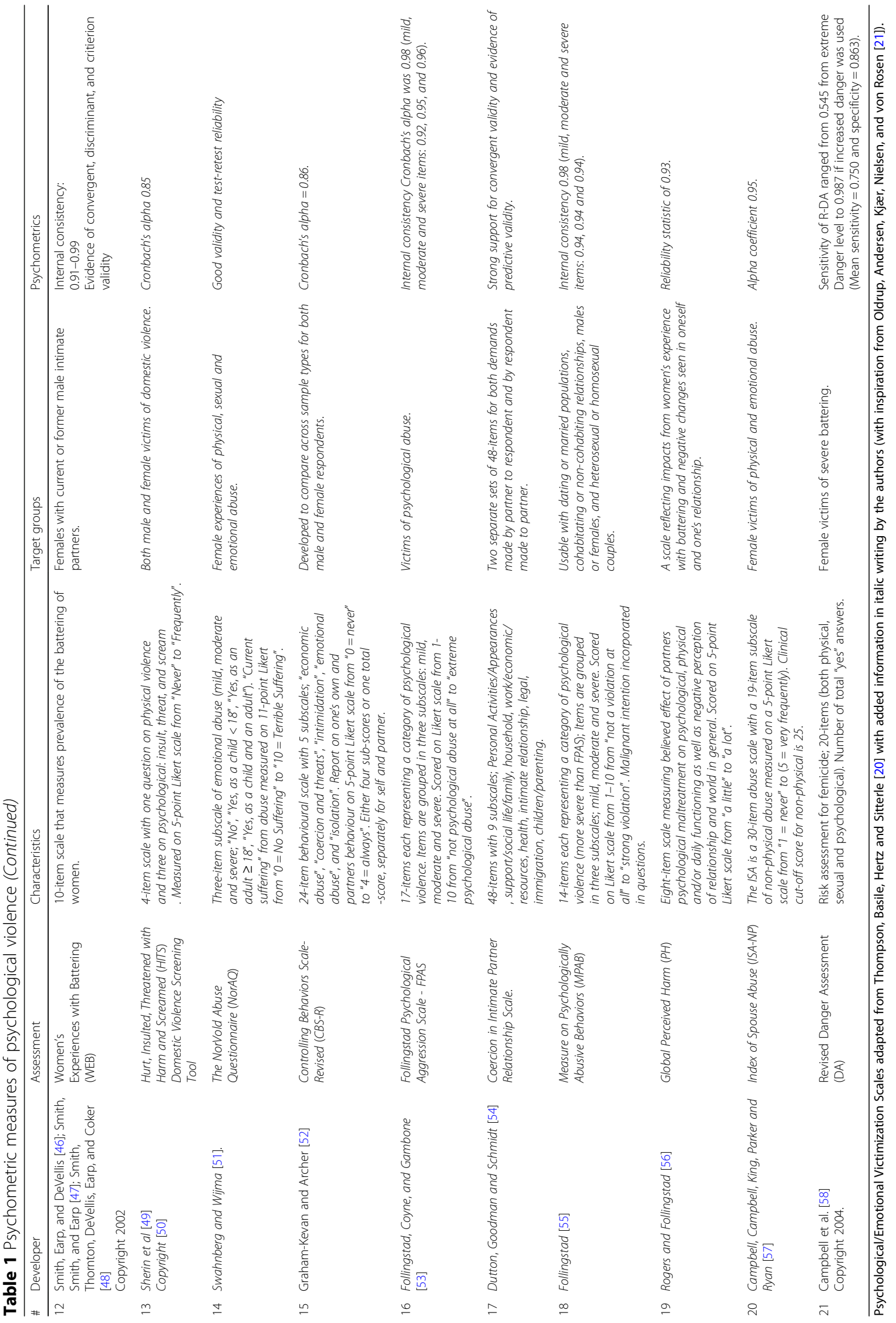


"type" of psychological violence produces the most harm on the victim's mental health [11].

In addition to problems with the conceptualization, a number of methodological challenges further characterize the field, e.g. sampling, design, scoring and gender bias [11]. Focusing on psychological aggression in lesbian, gay and bisexual individuals, Mason et al. [22] highlight the need for future research to clearly and consistently define psychological violence and separate it from other types of violence seeing that a more consistent definition will facilitate better comparisons across studies. The research group further stresses that scoring methods (e.g. frequency vs. dichotomous scoring) influence the magnitude of the effect size, which makes results difficult to compare. This challenge is further complicated by the use of selfadministered questionnaires that may lack systematic development [22]. Moreover, Follingstad [11] emphasizes the need to differentiate between samples (i.e. dating relationships and marital or long-term cohabiting relationships), seeing that dating relationships are characterized by quantitatively and qualitatively less psychological violence. Finally, the majority of assessment tools are developed to specifically measure female victimization of psychological violence, despite male victimization being reported at equally high rates in some studies [7]. Although fewer studies have focused on the effects on mental health among male victims of psychological violence, studies indicate that they too present symptoms of anxiety, depression and sleep disturbances [13, 23].

The aim of the present systematic review is to build on existing knowledge [13] concerning the effect of psychological violence on mental health, while evaluating the psychometric instruments used to assess psychological violence about how they conceptualize the phenomenon. To this day, most studies on IPV and mental health have pooled scores of physical, psychological and sexual IPV in their reporting [2, 4], making a distinction of the individual effect of psychological violence difficult. When directly examining the effect of psychological violence, the lack of a clear and consistent definition of psychological violence has made results difficult to compare [13, 22]. Consequently, important information is lost. The present study therefore aims to consolidate knowledge on psychological violence by evaluating whether frequency, severity or actual "type" of psychological aggression is associated with the most harm on mental health [11]. Methodological challenges will be considered by conducting quality assessments of all included studies, and results will control for the presence of physical and sexual violence (i.e. severity, duration and frequency). When possible, mediating and moderating factors will be evaluated, as well as potential gender differences. Based on the results, a discussion on assessment tools and methodological challenges will provide the grounds for recommendations concerning future research.

To sum up, the aim of this systematic review and meta-analysis is fourfold: (1) to estimate the individual effect of psychological violence on mental health (e.g. PTSD, depression and anxiety); (2) to estimate whether frequency, severity or actual "type" of violence is associated with the most harm; (3) to investigate gender, sampling and cultural differences through moderation analyses; and (4) to discuss the somewhat vague terminology and methodological challenges.

\section{Methods and design}

\section{Methods of review}

The present protocol has been written in accordance with the Preferred Reporting Items for Systematic reviews and Meta-Analyses (PRISMA) guidelines and is presented in accordance with the PRISMA-P checklist (Additional file 1). The protocol has further been registered in PROSPERO (\#CRD42018116026).

The systematic review will be conducted as an individual and dual process by two researchers $(\mathrm{SD}+\mathrm{DBH})$ in regard to screening, eligibility and inclusion. Screening will be done on a title basis, followed by an abstract and full-text basis. A third researcher (RK) from the team will be consulted to resolve issues regarding disagreement of eligibility and inclusion on a full-text basis. If sufficient data can be extracted, a random-effects metaanalysis will be conducted on the extracted data.

It is expected that not all studies report the recorded data on psychological violence, but rather cluster subtypes of IPV (i.e. physical, sexual and psychological violence). In such cases, the corresponding authors will be contacted and invited to share the raw data.

\section{Key definitions of the systematic review Intimate partner violence}

As proposed in a statistical definition by EIGE, IPV is defined as "any act of physical, sexual, psychological or economic violence that occurs between former or current spouses or partners, whether or not the perpetrator shares or has shared the same residence with the victim" [19] (p. 44). This definition has been proposed to aid the EU member states to collect and compare administrative data on violence against women in a standardized manner [19].

\section{Psychological violence}

A broad terminology for psychological violence is used in both scientific literature and in clinical practice (e.g. psychological violence, emotional abuse, coercion, psychological aggression). For clarity, this review will use the term psychological violence seeing that this definition links it directly to other types of IPV (e.g. physical or 
sexual violence), while emphasizing a core psychological aspect of harm in both the act of perpetration as well as the effect of victimization. The variation in terminology will shortly be addressed in the discussion.

In the current protocol, psychological violence will be defined by an overall definition combining that of both the WHO [1] (i.e. both emotional/psychological abuse and controlling behaviours) and the European Institute of Gender Equality [19]: "Any act or behaviour which causes psychological harm to the partner or former partner. Psychological violence can take the form of, among others, coercion, defamation, a verbal insult or harassment" [19] (p. 45), including belittling, constant humiliation, intimidation (e.g. destroying things), threats of harm, threats to take away children and/or isolating a person from family and friends; monitoring their movements; and restricting access to financial resources, employment, education or medical care [1] (p. 1). A broad definition will allow for studies using varying definitions to be included and will be assessed through subgroup analyses. The different definitions will be evaluated and discussed based on their effect on mental health.

\section{Mental health in this context}

As mentioned above, it has previously been argued that psychological violence alone cannot be characterized as a trauma [11]. However, recent studies have identified an association between psychological violence and PTSD [13]. Based on these findings, the authors consider psychological violence a potential traumatic event and wish to further examine the relationship between psychological violence and PTSD. Other mental health consequences identified are based on the National Institute for Health and Care Excellence (NICE) guidelines and comorbid mental health problems of PTSD [24], i.e. depression, anxiety, alcohol or drug abuse, suicidality, sexual problems, sleep problems, problems with concentration, somatization and functional problems (e.g. social, educational, or occupational) as well as feelings of shame and guilt.

\section{Search method}

A dual search will be conducted in the electronic databases PsycINFO, PubMed, EMBASE and Web of Science. Other methods used for identifying relevant research include reference checking and hand-searching of grey literature. Furthermore, the following scientific journals will be hand-searched: Journal of Interpersonal Violence and Journal of Violence and Victims.

\section{Criteria for including studies}

The review will include studies of psychological violence on mental health when controlling for other types of partner abuse. Hence, studies including an adult $(\geq 18$ years) population of victims of IPV (dating samples, national samples, clinical settings, etc.) that report on psychological violence specifically. Many studies are expected to include groups of comparison (e.g. non-abused or other types of abuse); however, comparisons are not required.

Furthermore, the review will only include peer-reviewed articles in English, German, Dutch or Scandinavian languages (i.e. Norwegian, Swedish or Danish).

\section{Criteria for excluding studies}

The review will exclude case studies, reviews, commentaries, editorials, letters to editorials, book chapters and other non-primary research articles.

\section{Search string}

See Table 2 for the PsycINFO search string.

\section{Main outcome of interest}

To investigate the independent effect of psychological violence on mental health. A meta-analysis will be performed to estimate the effect of psychological violence on PTSD, depression and anxiety. A narrative summary will present all related mental health problems as defined by the NICE guidelines [24].

Secondary outcomes of interest include the following:

- How does "type" of psychological violence affect mental health?

- How does frequency and severity affect mental health outcomes? (e.g. high frequency and/or low severity or low frequency and/or high severity).

- Are there potential gender differences in mental health consequences with regard to psychological violence?

- Will controlling for previous trauma affect the association between psychological violence and mental health?

- Does sample population differ in mental health consequences? (e.g. dating samples vs. treatment samples)

Finally, the systematic review aims to evaluate included psychometric tools measuring psychological violence and how potential variations in the conceptualization of psychological violence affects results.

\section{Data extraction}

Data will be extracted with help from Endnote and Covidence. Meta-analyses will be conducted using the programming language $R$. The authors will design a data extraction form. The form will include authors, year, sample size, population, country, age, gender, design, IPV assessment tool(s), mental health assessment, 
Table 2 Search String

\begin{tabular}{|c|c|}
\hline & Search algorithm \\
\hline 1. Population & $\begin{array}{l}\text { Exp } \\
\text { exp Intimate Partner Violence/ OR exp COUPLES/ OR exp Partner Abuse/ OR exp Domestic Violence/ OR exp DYADS/ } \\
\text { OR exp MARRIAGE/ OR exp HUSBANDS/ OR exp WIVES/ OR Wife OR *friend/ OR Girlfriend OR Boyfriend OR Dating OR } \\
\text { Domestic OR Partner }\end{array}$ \\
\hline 2. Exposure & $\begin{array}{l}\text { Keywords } \\
\text { Psychological Victimization OR Emotional Victimization OR Psychological Violence OR Psychological Abuse OR } \\
\text { Psychological Assault OR Aggression OR Psychological Aggression OR Emotional Aggression OR Emotional Abuse OR } \\
\text { Emotional Assault OR Emotional Violence OR Coercive Control OR Coercion OR Humiliation }\end{array}$ \\
\hline 3. Measures/scales & $\begin{array}{l}\text { Keywords } \\
\text { Abusive behaviour Inventory OR (Composite Abuse Scale OR CAS) OR Index of Psychological Abuse OR Measure of Wife } \\
\text { Abuse OR Multidimensional Measure of Emotional Abuse OR Partner Abuse Scale-Non Physical OR (Psychological Mal- } \\
\text { treatment of Women Inventory OR PMWI) OR (Revised Conflict Tactic Scale OR CTS2 OR CTS-2) OR Safe Dates - Psycho- } \\
\text { logical Abuse Victimization OR (Women's Experiences with Battering OR WEB) OR (Tool for Intimate Violence Screening } \\
\text { OR HITS) OR (NorVold Abuse Questionnaire OR NorAQ) OR (Controlling Behaviors Scale-Revised OR CBS-R) OR (Folling- } \\
\text { stad Psychological Aggression Scale OR FPAS) OR Yllo's Controlling Behavior Questions OR Coercion in Intimate Partner } \\
\text { Relationship Scale OR (Measure on Psychologically Abusive Behaviors OR MPAB) OR (Global Perceived Harm OR PH) OR } \\
\text { (Index of Spouse Abuse OR ISA-NP) OR (Danger Assessment OR DA) }\end{array}$ \\
\hline 4. & $2 O R 3$ \\
\hline $\begin{array}{l}\text { 5. Outcome (mental } \\
\text { health) }\end{array}$ & $\begin{array}{l}\text { Exp and keywords } \\
\text { exp Mental Health/ OR exp Emotional Trauma/ OR exp COMORBIDITY/ OR exp Posttraumatic Stress Disorder/ OR exp } \\
\text { MAJOR DEPRESSION/ OR exp "DEPRESSION (EMOTION)"/ OR exp ANXIETY DISORDERS/ OR exp ANXIETY/ OR exp } \\
\text { ALCOHOL ABUSE/ OR exp DRUG ABUSE/ OR exp ATTEMPTED SUICIDE/ OR exp SUICIDE/ OR Mental Health.mp. OR } \\
\text { Emotional Trauma.mp. OR COMORBIDITY.mp. OR COMOR*.mp. OR Posttraumatic Stress Disorder.mp. OR Post-traumatic } \\
\text { stress.mp. OR PTSD.mp. OR Posttraumatic stress symptoms.mp. OR Post-traumatic stress.mp. OR PTSS.mp. OR DEPRES- } \\
\text { SION.mp. OR Major Depression.mp. OR Depress*.mp. OR ANXIETY.mp. OR Anxiety DISORDERS.mp. OR Anxie*.mp. OR } \\
\text { Panic*.mp. OR Phobia.mp. OR Social Anxie*.mp. OR Substance Abuse.mp. OR ALCOHOL ABUSE.mp. OR DRUG ABUSE } \\
\text { SUICIDE.mp. OR ATTEMPTED SUICIDE.mp. OR Suicidal.mp. OR Shame.mp. OR Guilt.mp. OR Reduced Libido.mp. OR Sexual } \\
\text { Problems.mp. OR Social Functioning.mp. OR Educational Functioning.mp. OR Occupational Functioning.mp. OR Sleep } \\
\text { Problems.mp. OR Sleep Disorders.mp. OR Concentration Problems.mp. OR Job Loss.mp. OR Social Withdrawal.mp. OR } \\
\text { Social Isolation.mp. OR Somatization.mp. OR Somatic Complaints.mp. OR Chronic Pain.mp. OR Pain.mp. OR Poor } \\
\text { Health.mp. OR Medical Problems.mp. [mp=title, abstract, heading word, table of contents, key concepts, original title, } \\
\text { tests \& measures] }\end{array}$ \\
\hline 6. & 1 AND 4 AND 5 \\
\hline
\end{tabular}

primary outcome (effect size), secondary outcome(s), timeframe of assessment (lifetime or specified), scoring method(s), previous trauma and previous mental health problems. Data will be extracted by one reviewer (SD), and independently cross-checked by another reviewer $(\mathrm{DBH})$. Inconsistencies in data extraction will be resolved between the reviewers by referring to the source study until a consensus is reached.

\section{Quality assessment}

The "Quality Assessment Tool for Quantitative Studies" developed by the Effective Public Health Practice Project [25] will be used to assess the quality of the included studies. This is in line with previous research evaluating IPV psychometric tools [26]. Assessment will be based on six components: (1) selection bias, (2) study design, (3) confounders, (4) blinding, (5) data collection methods and (6) withdrawals and dropouts [24, 25]. Two researchers (SD and $\mathrm{DBH}$ ) will classify studies on three levels: weak, moderate and strong. If classifications are inconsistent a third researcher (RK) will be involved and classification will be discussed until consensus is reached. If possible, moderation analyses will compare studies of strong vs. weak quality.

\section{Plan for data synthesis}

As demonstrated above, studies on psychological violence are quite heterogeneous in regard to conceptualization, psychometrics, sampling, design, scoring, and so forth. Therefore, we will perform a random-effects meta-analysis, because we expect high heterogeneity in the included studies. The randomeffects meta-analysis assumes variance in effect across studies due to real differences in effect as well as by chance. The meta-analysis will help estimate the common effect of psychological violence on mental health (i.e. PTSD, depression and anxiety) by synthesizing individual results. If possible, moderation analyses will compare studies according to quality assessment and varying samples. The $I^{2}$ statistic will be used to test for heterogeneity, and as suggested, an $I^{2}$ statistic above $75 \%$ implies considerable heterogeneity, while an $I^{2}$ statistic below $40 \%$ is not considered to be a concern [27].

The included studies are expected to report effect sizes of varying types (i.e. correlation, regression, mean 
differences and association of categorical variables, e.g. odds ratio). For data synthesis, the reported effect sizes will be recoded into the same type of effect size using the programming language R. For this meta-analysis, we expect to perform a stepwise analysis according to the outcome of interest. For the main outcome of interest, as well as subgroup analyses, the meta-analysis will be conducted with effect sizes based on correlations. Additionally, the impact of the duration and frequency of psychological violence will be estimated by using meta-regression. The Metafor-package for the programming language $\mathrm{R}$ will be applied to conduct the metaanalysis [28].

\section{Discussion}

The present review will build on existing knowledge by statistically synthesizing results on the effect of psychological violence on mental health. The review will help consolidate knowledge on psychological violence by evaluating whether frequency, severity or actual "type" of psychological violence produces the most harm. This will strengthen our knowledge on psychological violence, and how best to assess and conceptualize the phenomenon.

While conducting the systematic review, we expect to encounter several limitations. The varying terminology and definitions of psychological violence will make results difficult to compare, just as the many and varying psychometrics tools will (Table 1). This is further complicated by the fact that many studies are likely to not even use validated instruments but instead base their results on a few self-constructed items. The review will aim to overcome these limitations by thorough quality assessment of the included studies with help from the Quality Assessment Tool for Quantitative Studies [25] and by performing relevant subgroup analyses. This does not only apply to psychological violence, but also mental health definitions and the psychometric instruments used to assess symptomatology. By critically examining the applied definitions and terminology as well as methodological challenges (e.g. sampling, design, scoring and gender bias) the review will serve as a status quo of the field and make grounds for future recommendations.

Finally, the systematic review is expected to have several clinical implications. We expect the meta-analysis to deepen our understanding of the different subtypes of psychological violence and how they independently interact with mental health outcomes. Likewise, we expect to give clarity on psychological violence and whether it should be understood as a traumatic event equal to other types of abuse (e.g. physical or sexual violence). Developing both preventive efforts and treatment programmes such information is important if we wish to directly target the needs of those affected by psychological violence and raise awareness to encourage both victims and perpetrators to seek help.

In research, this systematic review is expected to inspire researchers to clearly and consistently define psychological violence while carefully considering the psychometrics used to measure the phenomenon, as well as other methodological challenges. Moreover, we expect to get clarity on any potential gender bias.

On a final note, this work will hopefully inspire others to conduct similar research on children who grow up as either witnesses or victims of psychological violence.

\section{Additional file}

Additional file 1: PRISMA-P 2015 Checklist (DOCX $30 \mathrm{~kb})$

\section{Acknowledgements}

Not applicable.

\section{Authors' contributions}

The present protocol has been prepared and written by the corresponding author Sarah Dokkedahl, with contributions from all the co-authors. All authors have contributed to the development of the search string and Robin Kok contributed in the development of the plan for data synthesis. All authors have read and approved the final manuscript.

\section{Funding}

These materials have received financial support from The Danish Victims Fund. The execution, content, and results of the materials are the sole responsibly of the authors. The analysis and viewpoints that have been made evident from the materials belong to the authors and do not necessarily reflect the views of The Council of The Danish Victims Fund. Funding was granted to Sarah Dokkedahl.

Availability of data and materials

The datasets used and/or analysed during the current study are available from the corresponding author on reasonable request.

Ethics approval and consent to participate

Not applicable.

Consent for publication

Not applicable.

\section{Competing interests}

The authors declare that they have no competing interests.

\section{Author details}

'National Centre of Psychotraumatology, Department of Psychology at the University of Southern Denmark, 5230 Odense M, Denmark. ${ }^{2}$ Department of Psychology at the University of Southern Denmark, Odense, Denmark. ${ }^{3}$ Centre for Innovative Medical Technology, Odense University Hospital, Odense, Denmark. ${ }^{4}$ Centre for persons subjected to violence, Frederiksberg Hospital, Frederiksberg, Denmark.

Received: 6 December 2018 Accepted: 26 July 2019

Published online: 09 August 2019

\section{References}

1. World Health Organization. Understanding and addressing violence against women: 2012. Retrieved from: http://apps.who.int/iris/bitstream/handle/1 0665/77432/WHO_RHR_12.36_eng.pdf? sequence $=1$.

2. Golding JM. Intimate partner violence as a risk factor for mental disorders: A meta-analysis. J Fam Violence. 1999;14(2):99-132. 
3. Dutton MA, Green BL, Kaltman SI, Roesch DM, Zeffiro TA, Krause ED. Intimate partner violence, PTSD, and adverse health outcomes. J Interpers Violence. 2006;21(7):955-68.

4. Johnson DM, Zlotnick C, Perez S. The relative contribution of abuse severity and PTSD severity on the psychiatric and social morbidity of battered women in shelters. Behavior Therapy. 2008;39:232-41.

5. Devries KM, Mak JY, Bacchus $L J$, Child JC, Falder G, Petzold M, et al. Intimate partner violence and incident depressive symptoms and suicide attempts: A systematic review of longitudinal studies. PLOS Med. 2013;10(5):e1001439.

6. Pigeon WR, Cerulli CJD, Richards HMS, He H, Perlis M, Caine E. Sleep disturbances and their association with mental health among women exposed to intimate partner violence. J Women's Health. 2011;20(12):1923-9.

7. Black MC, Basile KC, Breiding MJ, Smith SG, Walters ML, Merrick MT, et al. The National Intimate Partner and Sexual Violence Survey (NISVS): 2010 summary report. Atlanta, GA: National Center for Injury Prevention and Control, Centers for Disease Control and. Prevention. 2011; Retrieved from: https://www.cdc.gov/violenceprevention/pdf/nisvs_report2010-a.pdf.

8. European Union Agency for Fundamental Rights (EUAFR). Violence against women, an EU-wide survey: Main results. Vienna: European Union Agency for Fundamental Rights; 2014. Retrieved from https:/fra.europa.eu/en/ publication/2014/violence-against-women-eu-wide-survey-main-results-report.

9. Aas $G$, Andersen T. Mishandlingsbestemmelsen - En evaluering av loven mot mishandling I nære relasjoner jf. Strl. § 219 (§ 282/283). Politihøgskolen, Oslo; 2017. Retrieved from: https://phs.brage.unit.no/phs-xmlui/handle/1125 $0 / 2427624$.

10. Home Office. Controlling or coercive behaviour in an intimate or family relationship - statutory guidance. framework. December, 2015; Retrieved from: https://assets.publishing.service.gov.uk/government/uploads/system/ uploads/attachment_data/file/482528/Controlling_or_coercive_ behaviour___statutory_guidance.pdf.

11. Follingstad DR. The impact of psychological aggression on women's mental health and behavior - the status of the field. Trauma, Violence, Abuse. 2009; 10(3):271-89.

12. American Psychiatric Association. Diagnostic and statistical manual of mental disorders: DSM-IV-TR. Washington, DC: American Psychiatric Association; 2000.

13. Lagdon S, Armour C. Stringer. Adult experiences of mental health outcomes as a result of intimate partner violence victimization: A systematic review. Eur J Psychotraumatol. 2014:5:24794.

14. Stark E. Coercive control. New York: Oxford University Press; 2007.

15. Stark E. Do violent acts equal abuse? Sex roles. 2010;62:201-11.

16. Johnson MP. Patriarchal terrorism and common couple violence. J Marriage Fam. 1995;57(2):283-94.

17. Johnson MP. A typology of domestic violence. Boston, MA: Northeastern University Press; 2008

18. Walby S, Towers J. Untangling the concept of coercive control: Theorizing domestic violent crime. Criminology Criminal Justice. 2018;18(1):7-28.

19. EIGE. Glossary of definitions of rape, femicide, and intimate partner violence; 2017. Retrieved from: https://eige.europa.eu/rdc/eige-publications/glossarydefinitions-rape-femicide-and-intimate-partner-violence

20. Thompson MP, Basile KC, Hertz MF, Sitterle D. Measuring intimate violence victimization and perpetration: A compendium of assessment tools. Atlanta (GA): Centers for Disease Control and Prevention, National Center Injury. Prev Control. 2006.

21. Oldrup, H., Andersen, S., Kjær. S., Nielsen, NH., von Rosen, CB. Psykiske, fysiske og sociale konsekvenser af psykisk vold i parforhold kortlægning af forskning. Lev Uden Vold. København; 2018. Retrieved from: https://levudenvold.dk/wp-content/uploads/2018/08/rapportkonsekvenser-af-psykisk-vold-web.pdf

22. Mason TB, Lewis RJ, Milletich RJ, Kelley ML, Minifie JB, Derlega VJ. Psychological aggression in lesbian, gay, and bisexual individuals' intimate relationships: A review of prevalence, correlates, and measurement issues. Aggress Violent Behav. 2014;19:219-34.

23. Próspero M. The effect of coercion in aggression and mental health among reciprocally violent couples. J Fam Violence. 2008;23:195-202.

24. National Collaborating Centre for Mental Health. Post-traumatic stress disorder - the management of ptsd in adults and children in primary and secondary care. National Clinical Practice Guidelines Number 26. The Roya College of Psychiatrists \& The British Psychological Society. Gaskell and the British Psychological Society. Cromwell Press Limited, Trowbridge, Wiltshire; 2005. Retrieved from https://www.ncbi.n/m.nih.gov/books/NBK56494/pdf/ Bookshelf_NBK56494.pd.
25. National Collaborating Centre for Methods of and Tools. Qualitative assessment tool for quantitative studies. Hamilton, ON: McMaster University; 2008. (Updated 03 October, 2017). Retrieved from: https://www.nccmt.ca/ knowledge-repositories/search/14.

26. Arkins B, Begley C, Higgins A. Measures for screening intimate partner violence: A systematic review. J Psychiatr Ment Health Nurs. 2016;23:217-35.

27. Cooper $\mathrm{H}$. Research synthesis and meta-analysis. $5^{\text {th }}$ Ed. Duke University: Sage Publications, Inc.; 2017.

28. Viechtbauer W. Conducting meta-analyses in $\mathrm{R}$ with the metafor package. J Stat Softw. 2010;36:3:1-48.

29. Shepard MF, Campbell JA. The Abusive Behavior Inventory: A measure of psychological and physical abuse. J Interpers Violence. 1992;7:291-305.

30. Hegarty K, Sheehan M, Schonfeld C. A multidimensional definition of partner abuse: Development and preliminary validation of the Composite Abuse Scale. J Fam Violence. 1999:14:399-415.

31. Hegarty K, Bush R, Sheehan M. The Composite Abuse Scale: Further development and assessment of reliability and validity of a multidimensional partner abuse measure in clinical settings. Violence Victims. 2005;20:529-47.

32. Sullivan CM, Bybee DI. Reducing violence using community-based advocacy for women with abusive partners. J Consult Clin Psychol. 1999:67:43-53.

33. Sullivan CM, Parisian JA, Davidson WS. Index of psychological abuse: Development of a measure. Poster presentation at the annual conference of the American Psychological Association. San Francisco (CA; 1991.

34. O'Leary KD. Psychological abuse: A variable deserving critical attention in domestic violence. Violence Victims. 1999:14:3-23.

35. Rodenburg FA, Fantuzzo JW. The Measure of Wife Abuse: Steps toward the development of a comprehensive assessment technique. J Fam Violence. 1993;8:203-28.

36. Murphy CM, Hoover SA. Measuring emotional abuse in dating relationships as a multifactorial construct. Violence Victims. 1999;14:39-53.

37. Murphy C, Hoover S, Taft C. The Multidimensional Measure of Emotional Abuse: Factor structure and subscale validity. Toronto: Association for the Advancement of Behavior Therapy; 1999.

38. Hudson WW. The WALMYR assessment scales scoring manual. Tallahassee (FL): WALMYR Publishing Company; 1997.

39. Sackett LA, Saunders DG. The impact of different forms of psychological abuse on battered women. Violence and Victims. 1999;14:105-77.

40. Tolman RM. The development of a measure of psychological maltreatment of women by their male partners. Violence Victims. 1989;4:159-77.

41. Tolman RM. The validation of the Psychological Maltreatment of Women Inventory. Violence Victims. 1999;14:25-37.

42. Straus MA, Hamby SL, Boney-McCoy S, Sugarman DB. The Revised Conflict Tactics Scale (CTS2): development and preliminary psychometric data. J Fam Iss. 1996;17:283-316.

43. Straus MA, Hamby SL, Warren WL. The Conflict Tactics Scale handbook. Los Angeles (CA: Western Psychological Services; 2003.

44. Foshee VA, Linder GF, Bauman KE, et al. The Safe Dates project: Theoretical basis, evaluation design, and selected baseline findings. Am J of Prev Med. 1996;12:39-47

45. Foshee VA, Bauman KE, Arriaga XB, Helms RW, Koch GG, Linder GF. An evaluation of Safe Dates, an adolescent dating violence program. Am J Public Health. 1998:88:45-50.

46. Smith PH, Earp JL, DeVellis R. Measuring battering: Development of the Women's Experiences with Battering (WEB) Scale. Women's. Womens Health. 1995;(1):273-88.

47. Smith PH, Smith JB, Earp JL. Beyond the measurement trap: A reconstructed conceptualized and measurement of woman battering. Psychology of Women Quarterly. 1999;23:177-93.

48. Smith PH, Thornton GE, DeVellis R, Earp J, Coker AL. A population-based study of the prevalence and distinctiveness of battering, physical assault, and sexual assault in intimate relationships. Violence Against Women. 2002; 8:1208-32.

49. Sherin KM, Sinacore JM, Li XQ, Zitter RE, Shakil AHITS. A short domestic violence screening tool for use in a family practice setting. Fam Med. 1999; 30(7):508-12.

50. Sherin KM, Sinacore JM, Li XQ, Zitter RE, Shakil A. HITS: a short domestic violence screening tool for use in a family practice setting. Fam Med. 1998; 30(7):508-12.

51. Swahnberg IMK, Wijma B. The NorVold Abuse Abuse Questionnaire (NorAQ) - a validation of new measures of emotional, physical, and sexual abuse, 
and abuse in the health care system among women. Eur J Public Health. 2003;13:361-6.

52. Graham-Kevan N, Archer J. Physical aggression and control in heterosexual relationships: The effect of sampling. Violence Victims. 2003;18(2):181-96.

53. Follingstad DR, Coyne S, Gambone L. A representative measure of psychological aggression and its severity. Violence Victims. 2005;20(1):25-38

54. Dutton, MA., Goodman, L., Schmidt, RJ. Development and validation of coercive control measure for intimate partner violence - final technical report. COSMOS Corporation: Bethesda, Maryland; 2005. Retrieved from: https://www.ncjrs.gov/pdffiles1/nij/grants/214438.pdf

55. Follingstad DR. A measure of psychological abuse normed on a nationally representative sample of adults. J Interpers Violence. 2010;26(6):1-21.

56. Rogers JM, Follingstad DR. Women's experience to psychological abuse: Does that experience predict mental health outcomes? J Fam Violence. 2014;29(6):595-611.

57. Campbell DW, Campbell J, King C, Parker B, Ryan J. Violence Victims. 1994; 9(3):259-74.

58. Campbell JC, Webster DW, Glass N. The danger assessment - validation of a lethality risk assessment instrument for intimate partner femicide. J Interpers Violence. 2009;24(4):653-74.

\section{Publisher's Note}

Springer Nature remains neutral with regard to jurisdictional claims in published maps and institutional affiliations.

Ready to submit your research? Choose BMC and benefit from:

- fast, convenient online submission

- thorough peer review by experienced researchers in your field

- rapid publication on acceptance

- support for research data, including large and complex data types

- gold Open Access which fosters wider collaboration and increased citations

- maximum visibility for your research: over $100 \mathrm{M}$ website views per year

At BMC, research is always in progress.

Learn more biomedcentral.com/submissions 